\title{
Diffusionen und Irritationen
}

\author{
Neue Studien zur Geschichte der Licht- und Strahlenforschung \\ Monika Dommann
}

Vera Dünkel 2016: Röntgenblick und Schattenbild. Genese und Ästhetik einer neuen Art von Bildern. Emsdetten/Berlin: Edition Imorde, Gebunden, 296 S., EUR 79.00, ISBN 978-3-9428-1035-7.

Christian Vogel 2015: Epistemischer Sinn und ästhetische Wirkung: Das Betrachten von Röntgenbildern im Schaukasten, 1896-1930. Fotogeschichte, 138 (Jg. 35), Themenheft: Fotografie und Medizin. Von der Glasplatte zur Simulation. S. 19-28, ISSN 07205260 .

Niklaus Ingold 2015: Lichtduschen. Geschichte einer Gesundheitstechnik, 1890-1975. Zürich: Chronos (=Interferenzen - Studien zur Kulturgeschichte der Technik 22, herausgegeben von David Gugerli), Broschur, 280 S., CHF 38.00/EUR 34.00, ISBN 978-3-0340-12768.

Alexander von Schwerin 2015: Strahlenforschung: Bio- und Risikopolitik der DFG, 1920-1970. Stuttgart: Franz Steiner Verlag (=Studien zur Geschichte der Deutschen Forschungsgemeinschaft 10), Kartoniert, 505 S., EUR 60.00, ISBN 978-3-515-10633-7.

Die deutsche Lyrikerin Claire Goll lobpreiste sie allesamt 1922, in ihrem Gedicht mit dem schlichten Titel "Zwanzigstes Jahrhundert". ${ }^{1}$ Die wissenschaftlichen und technischen Innovationen, welche den ingenieurwissenschaftlichen und physikalischen Labors des 19. Jahrhunderts entstammten und für das 20. Jahrhundert grundlegend wurden: etwa das Grammophon, die Röntgenstrahlen, die Kunstsonnen, die Benzmotoren, das Radium, das Kino, das Pianola, die Hochbahn. 
Was sollen uns grüne Teiche mit Weiden?

(Es ist zuviel Leid in der Welt um zu träumen!)

Was die gelben Punkte, die Sterne?

Jeden Abend um sieben

Gegen magische X-Strahlen

Und der Kunstsonne Violett? ${ }^{2}$

Golls Ode an die künstliche Natur war voll von fürchterlicher Faszination und faszinierter Furcht. Die Kunstsonnen, die Röntgenstrahlen und die Radioaktivität standen für eine künstliche, im Labor hergestellte zweite Natur. Sie stellten die Wahrnehmung in Frage und inspirierten Künstlerinnen und Künstler der Avantgarde. Sie polten die medizinische Diagnostik zur Bildwissenschaft um. Und sie wurden zum begehrten Objekt für gigantische militärische Big Science Projekte und ein Treiber staatlicher Forschungspolitik in den Weltkriegen und während des Kalten Kriegs. Als die Strahlenforschung am Ende des Kalten Kriegs im Zuge der Laborstudien und des practical turns in den Fokus der Wissenschaftsforschung und Wissensgeschichte geriet, konnten diese zwar auf Studien zur Geschichte der Röntgenstrahlen aufbauen, allerdings existieren bisher fast ausschließlich Entdeckungs-, Diffusions- und Disziplinengeschichten der Radiologie. In den Bibliotheken hatten sich jedoch reichhaltige Bildbände und Materialsammlungen angesammelt, welche den Strahleninteressierten die brachliegenden Schätze und das Potential für neue Fragestellungen vor Augen führten. ${ }^{3}$ Das Interesse an den Strahlen hatte sich nämlich verschoben: Es galt nicht mehr einer personen- und institutionszentrierten Perspektive und einer rein internalistischen, ideengeschichtlichen Herangehensweise, sondern dem Labor als sozialem Raum, der Materialität der Strahlen, den durchstrahlten Körpern, den veränderten Sehgewohnheiten und dem gesellschaftlichen Kontext. Das neue Interesse an den Strahlen in der Inkubationsphase von STS, wieder entdeckter Epistemologie, Körpergeschichte, Bild- und Medienwissenschaft führte zu einer Reihe von inspirierenden Studien, die zwischen 1980 und 2000 erschienen: technikhistorische Arbeiten, welche die materielle Kultur ins Zentrum stellten und in Bezug zur Sozialstruktur im Labor brachten, ${ }^{4}$ konstruktivistisch und bildwissenschaftlich motivierte Studien, welche sich den Anfängen der Bilddiagnostik in der Medizin zuwandten, ${ }^{5}$ alltagshistorische Arbeiten, die sich mit der Wahrnehmungsgeschichte der neuen Strahlen um die Jahrhundertwende beschäftigten, ${ }^{6}$ wissenshistorische Untersuchungen zum Wandel der Strahlenforschung nach Hiroshima, ${ }^{7}$ Analysen der sozialen Gebrauchsweisen der Röntgenbilder in Wissenschaft, Recht und Wirtschaft, ${ }^{8}$ und Auseinandersetzungen mit der Rezeption der Röntgenstrahlen in der Kunst. ${ }^{9}$ Insgesamt zeigten die vielen, auf bislang kaum beachteten Quellen basierenden Studi- 
en, dass die Herstellung der Strahlen in den Labors und die Diffusion der Technik in die Industrie, Kunst, Medizin und Naturwissenschaften von Zufällen geprägt war, undiszipliniert und kaum reguliert ablief und Effekte erzeugte, die sich nicht auf die bislang im Zentrum der Aufmerksamkeit stehenden Felder der Medizin und Naturwissenschaft beschränkten.

Dass das Interesse an diesem Forschungsfeld bis heute nicht nachgelassen hat, ist diesen multiplen Effekten der Labors zuzuordnen, denen sich die Forscherinnen und Forscher seit den 1980er Jahren, ganz der Devise einer lokalen und situierten Technikgestaltung und Wissensproduktion verpflichtet, dezidiert mikrohistorisch zugewandt hatten. Die vier neuen Studien, die hier zur Diskussion stehen, öffnen die Mikroperspektive des Labors. Durch das Prisma der Licht- und Strahlenforschung gelingen erhellende wissens-, bild- und medienhistorische Blicke auf das 20. Jahrhundert.

\section{Seherlebnisse}

Die Kunsthistorikerin Vera Dünkel hat für ihre an der Humboldt Universität zu Berlin verfasste Dissertation in zahlreichen Archiven und Publikationen gestöbert und eine bezaubernde Sammlung jener frühen Röntgenbilder ausgegraben, welche die Zeitgenossen um die Jahrhundertwende derart faszinierten. Das Buch ist von Andreas Trogisch und Heike Grebin subtil gestaltet worden. Besonders reizvoll ist das Blättern von der Rückseite her: Hinter einem transparenten Schutzumschlag schimmert die Fotografie eines an „Monsieur Röntgen“ in Würzburg adressierten und mit einem Siegel verschnürten Pakets, wobei auch die nächste Seite, ein Röntgenbild einer Schachtel mit Taschenuhr, durchschimmert. ${ }^{10}$ Vera Dünkels Buchdeckel ist auch eine Nachstellung der Spielereien und Sehspektakel im Fin du Siècle. Die beiden überlagerten Bilder entstammen einer Bildkomposition, die in der Druckschrift des Physikers Charles-Edouard Guillaume aus dem Jahr 1896 über die unbekannten X-Strahlen abgedruckt ist. Das Bild war in Guillaumes Buch als Frontispiz prominent hinter einem Transparent platziert worden. Sein Status (und darin liegt ein Grund für die bis heute andauernde Anziehungskraft der frühen Röntgenbilder) bleibt unsicher: Ist das Paket verschickt worden, wie der Poststempel nahelegt? Oder ist es eigens für die Aufnahme drapiert und gar nie verschickt worden? Ist es ein Witz? Was wird überhaupt auf dem Bild dargestellt? Welche grauschwarzen Schatten sind der Kiste geschuldet und welche dem Inhalt? Auch wenn die Bildlegende die Ambivalenz der Darstellung unterschiedlicher Dichteverhältnisse verminderte (Holzkiste mit Taschenuhr und ihrer Kette), war es gerade die Bedeutungsoffenheit der Bilder, welche ihre Anschlussfähig- 
keit und ihren Erfolg in unterschiedlichen Kontexten unterstützten und sie zwischen Kunst und Wissenschaft mäandrieren ließen.

Die Transparenz der neuen Art von Bildern (wie sie von Vera Dünkel in Anlehnung an Wilhelm Conrad Röntgens Diktum „einer neuen Art von Strahlen“ genannt werden) ließ Unsichtbares gleichsam durchscheinen und deckte Verborgenes auf. Die Bilder irritierten, weil die dargestellten Objekte in eine enträumlichte Fläche transformiert wurden. Doch anders als die gleichzeitig entwickelte Kinematographie war die ästhetische Karriere der Röntgenbilder bloß von kurzer Dauer und der beschränkten Variationsmöglichkeiten der Röntgeneffekte geschuldet. Vera Dünkel erklärt diesen Unterschied damit, dass die frühen Röntgenbilder zwar mit etablierten Bildtraditionen brachen, jedoch in Bezug auf die abgebildeten Motive historisch tief verwurzelt waren, indem sie den drei Topoi der neuzeitlichen Kunstkammern (naturalia, artificialia und curiosita) entsprachen. ${ }^{11}$ Dünkel sieht in der intensiven Beschäftigung mit Röntgenbildern nach der Jahrhundertwende (etwa durch den Atlasmacher Rudolf Grashey) ${ }^{12}$ auch eine Bildwissenschaft avant la lettre, genauso, so wäre zu ergänzen, wie die Bildwissenschaft der Jahrtausendwende und ihre Zuwendung zu den Produktionsbedingungen als ein Effekt des Aufstiegs digitaler Bilder interpretiert werden könnte. Auch wenn einige der abgebildeten Bilder bereits in anderen Publikationen dokumentiert und analysiert worden sind, besticht Dünkels Buch durch die hervorragenden Reproduktionen in Originalgröße und exzellenter Druckqualität. Vera Dünkel zeigt überdies anschaulich, dass es die medialen Dispositive waren (etwa Reproduktion und Multiplikation sowie Stereoskopie), welche den Bildern zu großer Aufmerksamkeit verhalfen.

Auch die Studien des Wissenschaftshistorikers Christian Vogel unterstützen dieses medienwissenschaftliche Apriori der Signifikanz der materiellen Bildträger. In einem in der Zeitschrift Fotogeschichte veröffentlichten Aufsatz rückt er das Augenmerk auf die von Medizinern entwickelten elektrisch beleuchteten Schaukästen, welche dem Wunsch der Ärzte nach einem Medium entsprachen, das die feinen Details der Bilder akkurater zu wiedergeben versprach als die durch das fotografische Verfahren erzeugten Negativkopien. Aus bild- und medienwissenschaftlicher Perspektive ist es interessant, dass zur selben Zeit, als in der Kunst die Funktion des Rahmens an Bedeutung verlor, dieser in der Radiologie bei der Bildkritik als Sehhilfe wichtig wurde. Röntgenbildausschnitte konnten mittels der Rahmung abgesondert von der Umwelt in einer gewissen Geschlossenheit studiert werden. Insbesondere die großen Schaukästen eröffneten einen neuen epistemischen Blick durch unendlich viele Bildcollagen, welche durch Serialität eine Vergleichbarkeit der Bilder ermöglichten. 
Man kann sich der Magie der von Dünkel und Vogel präsentierten Röntgenbilder auch heute kaum entziehen. Die Röntgenbilder mögen zwar eine medizinische Revolution ausgelöst haben, doch sind sie bis heute eine Quelle für ästhetische Erfahrungen geblieben. Was ist denn Kunst anderes als ein sinnliches Eintauchen in verstörende Welten?

\section{Lichtduschen}

Der Historiker Niklaus Ingold ist in seiner an der Universität Zürich entstandenen Dissertation der Geschichte des von Claire Goll in ihrem Gedicht ebenfalls erwähnten Ultraviolettlichtes und ihrer Anwendungen zu Forschungs- und Heilzwecken nachgegangen. Die auf dem Buchcover abgebildete weiße Kleinfamilie (mit durchtrainierten Körpern in Badekleidung und Schutzbrillen, im modernen Wohnzimmer posierend, auf einem Kunststoffboden, vor einem Nierentisch mit einer Ultraviolettlampe) zeigt eine technoide Szenerie aus der Zeit nach dem Zweiten Weltkrieg, in der Körperkult, Fitness, Kosmetik und Gesundheit vereint sind. Mit dem Versprechen auf Gesundheit war das Naturlicht im Labor erzeugt und gezähmt worden. Es konnte nun mittels massenproduzierter Lampen nach Lust und Laune konsumiert werden. Es ist die große Stärke dieser erfrischend pointiert verfassten Promotionsschrift, dass sie die Geschichte von Gesundheit exemplarisch am Beispiel des Ultraviolettlichtes als ein Zusammenspiel von Lebensreformbewegung, Laborforschung, unternehmerischer Initiativen und Hygienepolitik begreift. Durch die Elektrifizierung der Labors, Arztpraxen und Sanatorien im ausgehenden 19. Jahrhundert entstand an dezentralisierten Orten eine regelrechte Lichtbegeisterung. Die hoch technisierte wissenschaftliche Strahlenforschung in den 1920er Jahren, die bald zum Lieblingskind der Forschungspolitik avancierte, konnte auf dieser breiten gesellschaftlichen Faszination aufbauen.

Im 19. Jahrhundert waren die verstreuten Bemühungen, die diesen Lichthype beförderten, mit drei Namen verknüpft: Dem amerikanischen Tausendsassa John Harvey Kellogg, der einen mit elektrischen Glühbirnen beheizten Schwitzkasten zur Beschleunigung von Stoffwechselvorgängen entwickelte. Dem deutschen Chemiker und Unternehmer Willibald Gebhardt, der Kelloggs fototherapeutischen Apparat auf der Weltausstellung 1893 in Chicago entdeckte, nach Europa brachte, mit physiologischem Wissen aus dem Labor und einer Bogenlampe verband und in die Kuranstalten einführte. Und dem dänischen Physiologen Niels R. Finsen, der zusammen mit dem Arzt Axel Reyn einen neuartigen, mit einer Linse versehenen Apparat konstruierte, der das Behandlungspotential der Lichtkur erwei- 
terte (etwa bei Hauttuberkulose) und damit den Weg zu therapeutischen Verfahren und einem boomenden Apparatemarkt ebnete.

In den Sanatorien, jenen Sozialbiotopen zur Genesung kranker Körper (die als Lichtluftgeschöpfe verstanden wurden), wurden die Licht- und Sonnenbadhallen in den 1920er Jahren in das Kurprogramm integriert. Die Lichtbadhallen der Kurhäuser und der Strahlenabteilungen von Krankenhäusern ermöglichten „Massenbestrahlungen“ und entsprachen damit den Forderungen der Krankenkassen und ihrem Rationalisierungskalkül, gleichzeitig mehrere Personen kostengünstig zu besonnen.

Die Erforschung des Ultraviolettlichtes, seiner physiologischen Wirkungen (etwa der Einfluss auf die Vitamin-D-Bildung und Leistungssteigerung im Sport und Militär) und die Entwicklung von Standards vermochten im Verbund mit den Röntgenstrahlen in den 1920er Jahren ein neues interdisziplinäres Forschungsfeld zu bündeln, das sich alsbald mit eigenen Zeitschriften und staatlichen Forschungsbudgets profilierte und auch dem Militär und den Machthabern des Nationalsozialismus als Allianzpartner anbot. Die Strahlenforschung im Nationalsozialismus war allerdings vom Brain-Drain der jüdischen Forscher*innen betroffen. Die erzwungene Migration bot jedoch Opportunisten wie dem Arbeitsphysiologen Gunther Lehmann, dem Biophysiker Boris Rajewsky und dem Pädiater Bernhard de Rudder neue Führungspositionen in den staatlichen Forschungsanstalten des Regimes. Doch auch diese Anbiederung an die Diktatur konnte den Anfang vom Ende des Booms der Lichtforschung nicht aufhalten: Die Behandlung der Rachitis hatte bereits in den 1920er Jahren Konkurrenz durch Produkte der Pharmaindustrie erhalten. Anfang der 1950er Jahre waren nun auch Medikamente zur Behandlung der Tuberkulose auf dem Markt erhältlich. Die Lichtforschung galt deshalb nicht mehr als jenes dynamische Forschungsfeld, das von den 1920er bis in die 1940er Jahre ökonomisches und soziales Kapitel zu bündeln vermochte.

Ganz anders verlief die Karriere der Quarzlampen $\mathrm{GmbH}$, des nach der Jahrhundertwende gegründeten Marktführers von Quarzlampen: Die Produkte der Quarzlampengesellschaft hatten mit Unterstützung des Amtes für Volksgesundheit der NSDAP in Großbestrahlungsanlagen für Bergbauarbeiter der Firma Krupp in den 1930er Jahren Anwendung gefunden. Sie konnten sich auch nach dem Krieg im Rahmen eines neuen umfassenden Gesundheitsbegriffes der Weltgesundheitsorganisation neu als Anbieter von Techniken zur Stärkung der Gesundheit, Arbeitskraft und des Leistungsvermögens profilieren und in den Boomjahrzehnten der Nachkriegszeit mit den Elektrosonnen auch den Massenkonsummarkt bedienen. Die Elektrosonnen verhalfen der weißen Mittelklasse zur modischen und verschönernden Bräune, eine Bräune, die seit den 1970er Jahren aller- 
dings bei den Dermatologen in Verruf geriet. Nun galt es, die biologisch wirksamen UVB Strahlen durch Cremes und Scheiben herauszufiltern.

Ingolds durch hilfreiche Kapitelzusammenfassungen auch für schnelle Leser"innen leicht lesbare Studie legt somit eine historische Beschreibung der gesellschaftlichen Diffusionen des im Labor des ausgehenden 19. Jahrhunderts erzeugten Lichts vor und damit eine Geschichte der von Karin Knorr Cetina als „Laboratisierung durch und durch“ bezeichneten „Manufaktur von Natur".13 Waren diese Prozesse in den Skizzen der soziologischen Labortheorien der 1980er und 1990er Jahre noch eine abstrakte Skizze geblieben, kann Ingold anschaulich zeigen, wie ein Wille zum Wissen, Geschäftssinn, Machtkalkül und das kollektive und individuelle Begehren nach Schönheit wandelnde Allianzen eingingen.

\section{Strahlenforschung}

Die an der Universität Braunschweig und am Max-Planck-Institut für Wissenschaftsgeschichte entstandene Habilitationsschrift des Wissenschaftshistorikers Alexander von Schwerin setzt bei der von Niklaus Ingold in seiner Studien bloß kursorisch gestreiften, vom Staat großzügig unterstützten Strahlenforschung in Deutschland während der 1920er Jahre an und verfolgt diese bis 1970. Durch das Prisma der Deutschen Forschungsgemeinschaft (DFG) betrachtet, gelingt der Studie eine dringend wünschenswerte stärkere Rückbindung der Wissensgeschichte an die Politikgeschichte, als dies bislang der Fall war. Schwerin versteht die Strahlen als zutiefst politischen Untersuchungsgegenstand und räumt dabei auch gleich mit zwei dominanten Selbstbeschreibungen der DFG auf: Erstens dem Mythos der Autonomie von Forschung, die durch fremde Steuerungsbestrebungen bedroht wird. Und zweitens dem Mythos der wissenschaftlichen Selbstverwaltung, das heißt der Autonomie der Forschungsorganisationen gegenüber der Politik.

Die Manufaktur von Strahlen im Labor erzeugte politische Begehrlichkeiten, die von Alexander von Schwerin mit Rückgriff auf Michel Foucault als biopolitische Maßnahmen interpretiert werden, die direkt auf den Körper der Bevölkerung zielten. Die nach dem Ersten Weltkrieg 1920 gegründete Notgemeinschaft der Deutschen Wissenschaft unterstützte die Strahlenforschung zunächst bei der Herstellung und dem Verleih von Instrumenten und Apparaten und förderte somit die Technisierung und Experimentalisierung der Strahlenforschung - und zwar immer mit dem Ziel eines gesellschaftlichen Nutzens vor Augen, etwa in der Metallurgie oder Zell- und Textilfaserindustrie. Wie stark die Eingriffe der DFG in die For- 
schung waren, zeigte sich ab Mitte der 1920er Jahre auch in der Maßgabe von Leitlinien zur Förderung der Strahlenkunde, die ausdrücklich über die Grenzen der medizinischen Forschung herausreichen sollte. Die Planungskommission interessierte sich zunehmend für das UV-Licht, die Sonnenstrahlen und die kosmischen Strahlen und machte sich dabei für eine Standardisierung der boomenden Lichttherapien und die Entwicklung einer naturwissenschaftlichen, sprich physiologischen Methodik im Rahmen einer auf technische Operationalisierung ausgerichteten Gesundheitspflege stark. Zudem engagierte sich die DFG in der risikopolitischen Absicherung der durch Strahlenforschung induzierten Strahlengefahr: Eugenische Vorsorge und Genetik wurden zu einem Stützpfeiler der zunehmend bevölkerungshygienisch ausgerichteten Forschungspolitik der DFG.

Besonders eindrücklich ist die minutiöse Rekonstruktion der Verwicklungen der durch die DFG geförderten Strahlenforschung mit dem nationalsozialistischen Herrschaftssystem. Die NS Zeit erwies sich für diesen Forschungszweig gewissermaßen als Eldorado: Rassenhygienisch und genetisch gleichermaßen motiviert gerieten nach 1930 die „Erbschädigung durch Bestrahlung“, die Mutationen der Zellen und damit ein biopolitischer Blick auf die Bevölkerung in den Fokus der Forschungspolitik. Dabei folgten die Forschungspolitiker und die Forscher allerdings keinem Plan: Die neuen Schwerpunkte entstanden im Rahmen jenes Schmelztiegels zwischen Physik und Genetik, der durch die DFG bestens alimentiert worden war. Indem sie die technische Ausstattung der Institute unterstützte und Risikopolitik zuoberst auf ihre Agenda setzte, finanzierte die DFG letztlich während der 1930er Jahre einen großen Teil der strahlengenetischen Arbeiten. Wenn die Strahlenforscher sich in intensiven Auseinandersetzung zwischen verschiedenen Modellen der Strahlenwirkung verwickelten, betrieben sie nicht bloß Grundlagenforschung, sondern folgten damit, so Schwerin, auch forschungspolitischen und staatspolitischen Agenden des nationalsozialistischen Staates. Auch wenn die DFG bei der rassenpolitischen Wendung der Strahlenforschung im Rahmen von Zwangsumsiedlung, Sterilisation und Vernichtung keine tragende Rolle spielte, hatte sie dennoch durch die Erstellung von Expertise in der Bioklimatologie und mit ihrer Technisierungspolitik indirekt dafür den Boden bereitet.

Im Zentrum eines neuen Regulierungsregimes der DFG seit Mitte der 1930er Jahre stand die Messung, Prüfung und Regulierung der Strahlen im Rahmen eines Strahlenschutzes. Die Prüfwissenschaft der deutschen Atomplanungen hätte sich kaum von jener im Umkreis des ManhattanProjektes in den USA unterschieden - außer dass in Deutschland Biophysiker und Genetiker statt Radiologen wie in den USA die Risikoforschung bestimmten. Die biophysikalische Strahlenforschung erwies sich nach dem Abwurf der Atombomben über Nagasaki und Hiroshima als ungebrochen 
attraktiv. Einige NS-Strahlenforscher aus dem Umfeld des Kaiser-WilhelmInstitutes für Biophysik wurden von den USA denn auch für geheime militärische Projekte abgeworben. Auch hinsichtlich der Nachkriegsgeschichte der DFG sieht Schwerin in Bezug auf die Strahlenforschung viel Kontinuität, indem die biophysikalische Risikoforschung der 1930er und 1940er Jahre einfach fortgesetzt wurde. Strahlenschutz und Isotopenlabors standen nun im Zentrum der Forschungsförderung. Anfang der 1960er Jahre entbrannte ein Streit um Ressourcen und Zielrichtungen der Radiobiologie innerhalb der Scientific Community. Alexander von Schwerin betont, dass sich hinter diesen forschungs- und forschungspolitischen Differenzen auch ein sozialer Konflikt verbarg, indem die jüngere Generation ein Revival der überkommenen Eugenik befürchtete und sich stattdessen dem unverbrauchten Feld der molekulargenetischen Entschlüsselung des Lebens widmen wollte.

Die atomare Option der Bundesrepublik und die Förderung der atomaren Energie veränderten die Forschungspolitik nochmals radikal: Mit dem Bundesministerium für Atomfragen trat Mitte der 1950er Jahre ein neuer mächtiger Akteur auf das Wissenschaftsparkett, welcher langfristige Projekte in der Strahlenbiologie mit gigantischen Budgets alimentierte. Die DFG operierte, so Schwerins Verdikt, geschickt als Schaltstelle einer Risikopolitik, welche unter dem Primat der Gesundheitsforschung immer auch eine Forschung darüber war, in welchem Ausmaß mit den Strahlen aus dem Labor auch Krankheit und Tod in Kauf genommen werden mussten. Dies unter der Annahme eines Durchschnittsmenschen, der mithilfe statistisch ermittelter Grenzwerte die Sterblichkeitsrate in der Bevölkerung bestimmte.

Alexander von Schwerin hat eine materialreiche und minutiöse Geschichte der Strahlenforschung der DFG vorgelegt, die auf sorgfältiger Archivforschung (unter anderem im Bundesarchiv, im Archiv der DFG, dem Archiv der Max-Planck-Gesellschaft, einigen Universitäts- und Firmenarchiven sowie zahlreichen Nachlässen aus Archiven in Deutschland und den USA) beruht. Auch wenn die thesengeleitete Studie für Nichtspezialisten zuweilen etwas unübersichtlich daherkommt, hat Schwerin mit dieser grundlegenden Arbeit gezeigt, dass Wissenschaftsgeschichte weit mehr ist als eine Mikrogeschichte von Labors, sondern gleichzeitig auch eine Politikgeschichte von Machtverhältnissen des 20. Jahrhunderts.

Die vier besprochenen Arbeiten sprechen in mehrfacher Art und Weise zueinander. Sie zeigen eindrücklich, dass aus den vielen Forschungsinitiativen, die in den 1980er Jahren zwischen den natur-, geistes- und sozialwissenschaftlichen Disziplinen entstanden sind und das Wissen, die Technik, die Körper, die Bilder und die Medien einer Revision unterworfen hatten, inzwischen eine neue Generation von transdisziplinär forschenden 
Historiker*innen hervorgegangen ist, die an einer allgemeinen Geschichte des 20. Jahrhunderts mitschreibt, welche der Verwissenschaftlichung und Technisierung dieses Jahrhunderts adäquat Rechnung trägt. Sie sind auch ein Indiz dafür, wie prägend die theoretischen Impulse (etwa Wissen im Kontext, Akteur-Netzwerk-Theorie und Experimentalsystem) der letzten zwei Dekaden des 20. Jahrhunderts gegenwärtig nach wie vor sind. Fast fragt man sich da bang und hoffnungserfüllt zugleich: Wie wird die Geschichtsschreibung nach Michel Foucault, Bruno Latour, Hans-Jörg Rheinberger, W. J. T. Mitchell, Horst Bredekamp und wie sie alle heißen, wohl vorgehen? Welche Fragen wird sie antreiben? Dass sie dabei zurzeit die Politik, die Wirtschaft und die Medien stärker ins Zentrum stellt als noch in den 1980er Jahren, hat sich in den besprochenen Studien deutlich gezeigt. Vielleicht sollte sie einfach so weitermachen wie bisher? Den Akteuren und Dingen folgen, auf die Geldströme achten und die mediale Verfasstheit der Daten auf dem Weg durch die Gesellschaft? Doch sie wird, so hoffe ich doch auch, die theoretischen Impulse der letzten zwei Jahrzehnte des 20. Jahrhunderts nicht zu Schablone verkommen lassen und wieder vermehrt feilen an der Weiterentwicklung eines Werkzeugkastens einer künftigen Geschichtswissenschaft, deren künftige Fragen wir noch nicht kennen können.

\section{Anmerkungen}

1 Goll (1922), S. 7-8.

2 Goll (1922), S. 7.

3 Glasser (1931); Pallardy et al. (1989); Eisenberg (1992).

4 Vgl. z. B. Arns (1997); Blum (2000); Hessenbruch (2000a): Calibration and Work in the $\mathrm{X}$-Ray Economy.

5 Pasveer (1992); Cartwright (1995); Dommann (2003).

6 Hessenbruch (2000b): Science as Public Sphere.

7 Herzig (1999).

8 Golan (1998); Duffin \& Hayter (2000).

9 Henderson (1988); Geimer (2000), S. 119-135.

10 Vgl. dazu: Dünkel (2016): S. 104-105.

11 Dünkel (2016), S. 191.

12 Grashey (1905), (1908).

13 Knorr Cetina (1994), S. 112-113. 


\section{Literatur}

Arns, Robert G, 1997. The High-Vacuum X-Ray Tube. Technological Change in Social Context. Technology and Culture, 38, 852-890.

Blum, Martina, 2000. Die Entwicklung der Röntgenglühkathodenröhre. Lilienfeld, Coolidge und ihr Verhältnis zur Wissenschaft. In: Ivo Schneider, Helmuth Trischler und Ulrich Wengenroth, Hg., Oszillationen. Naturwissenschaftler und Ingenieure zwischen Forschung und Markt. München: Oldenbourg, 211-237.

Cartwright, Lisa, 1995. Screening the Body. Tracing Medicine's Visual Culture. Minneapolis, London: University of Minnesota Press.

Dommann, Monika, 2003. Durchsicht, Einsicht, Vorsicht. Eine Geschichte der Röntgenstrahlen, 1896-1963. Zürich: Chronos.

Duffin, Jacalyn und Charles R. R. Hayter, 2000. Baring the Sole. The Rise and Fall of the Shoe-Fitting Fluoroscope. Isis, 91, 260-282.

Dünkel, Vera, 2016. Röntgenblick und Schattenbild. Genese und Ästhetik einer neuen Art von Bildern. Emsdetten/Berlin: Edition Imorde.

Eisenberg, Ronald Lee, 1992. Radiology. An Illustrated History. St. Louis, MO: Mosby Year Book.

Geimer, Peter, 2000. Noise or Nature? Photography of the Invisible around 1900. In: Nowotny, Helga/Weiss, Martina, Hg., Shifting Boundaries of the Real: Making the Invisible Visible. Zürich: Vdf, 119-135.

Glasser, Otto, 1931. Wilhelm Conrad Röntgen und die Entdeckung der Röntgenstrahlen. Berlin: J. Springer.

Golan, Tal, 1998. The Authority of Shadows: The Legal Embrace of the X-Ray. In: Historical Reflections, 24/3, 437-458.

Goll, Claire, 1922. Zwanzigstes Jahrhundert. In: Lyrische Films. Gedichte. Basel/Leipzig: Rhein-Verlag, 7-8.

Grashey, Rudolf, 1905. Fehlerquellen und diagnostische Schwierigkeiten beim Röntgenverfahren. In: Münchner med. Wochenschrift, LII, 17, 807-810.

Grashey, Rudolf, 1908. Atlas chirurgisch-pathologischer Röntgenbilder. München: J. F. Lehmann.

Henderson, Linda Dalrymple, 1988. X-Rays and the Quest for Invisible Reality in the Art of Kupka, Duchamp and the Cubists. Art Journal, 47, 323-339.

Herzig, Rebecca, 1999. Removing Roots. "North American Hiroshima Maidens" and the $\mathrm{X}-$ Ray. Technology and Culture, 40, 723-745.

Hessenbruch, Arne, 2000a. Calibration and Work in the X-Ray Economy, 1896-1928. Social Studies of Science, 30/3, 397-420.

Hessenbruch, Arne, 2000b. Science as Public Sphere. X-Rays between Spiritism and Physics. In: Goschler, Constantin, Hg., Wissenschaft und Öffentlichkeit in Berlin, 1870-1930. Stuttgart: Steiner, 89-126.

Knorr Cetina, Karin, 1994. Die Manufaktur der Natur oder: Die alterierten Naturen der Naturwissenschaft. In: Wilke, Joachim, Hg., Zum Naturbegriff der Gegenwart, Band 1. Stuttgart-Bad Cannstatt: Frommann-Holzboog, 95-114.

Pallardy, Guy, Marie-José Pallardy, und Auguste Wackenheim, 1989. Histoire illustrée de la radiologie. Paris: R. Dacosta.

Pasveer, Bernike, 1992. Shadows of Knowledge. Making a Representation Practice in Medicine: X-ray Pictures and Pulmonary Tuberculosis 1895-1930. Amsterdam: Universität Amsterdam. 
Monika Dommann

Historisches Seminar

Universität Zürich

Karl Schmid-Strasse 4

8006 Zürich

Schweiz

monika.dommann@hist.uzh.ch 